\title{
TM-Algebra - An Introduction
}

\author{
K.Megalai \\ Department of Mathematics \\ Bannari Amman Institute of Technology \\ Tamil Nadu. India
}

\author{
Dr.A.Tamilarasi \\ Kongu Engineering College \\ Perundurai, Erode Dist. \\ Tamil Nadu, India
}

\begin{abstract}
In this paper, a new notion, called TM-algebra, which is a generalization of the idea of $\mathrm{Q} / \mathrm{BCH} / \mathrm{BCI} / \mathrm{BCK} / \mathrm{BCC}$-algebra, is introduced. Some theorems discussed in Q-and BCK algebras are generalized. Definition of TM-algebra along with various propositions are stipulated and presented with their respective proofs. The relation between TM-algebra and other algebra has been investigated and detailed in the paper. Also ideal, p-radical, p-semi simple are discussed .
\end{abstract}

Mathematics Subject Classification: 06F35, 03G25

\section{Keywords}

TM-Algebra, ideal, p-radical, p-semi simple.

\section{INTRODUCTION}

J.Neggers and Kim (see [1] ) introduced the notion of d-algebras which is a generalization of BCK- algebras. Also Joseph Neggers, Sun Shin Ahn, and Hee Sik Kim introduced Qalgebras[2], which is a generalization of $\mathrm{BCH} / \mathrm{BC} / \mathrm{BCK}-$ algebras, and generalized some theorems from the theory of BCIalgebras. Imai and Iseki introduced two classes of abstract algebras BCI-algebras and BCK-algebras [3, 4]). It is known that the class of BCK-algebras is a proper subclass of the class of BCI- algebra. In [5, 6] $\mathrm{Hu}$ and $\mathrm{Li}$ introduced a wide class of abstract algebra namely BCH- algebras., At the same time, Jun, Roh, and Kim [7] introduced a new notion called BH-algebra, which is a generalization of BCH / BCI / BCK-algebra. In this paper a new notion, called TM-algebra, which is a generalization of $\mathrm{BCH} / \mathrm{BCI} / \mathrm{BCK} / \mathrm{Q}-$ algebras, is introduced and some theorems of BCI/ BCK/Q-algebras are generalized..

\section{TM-ALGEBRA}

\subsection{Definition}

A TM-algebra $(\mathrm{X}, *, 0)$ is a non-empty set $\mathrm{X}$ with a constant 0 and a binary operation $*$ satisfying the following axioms :

(i) $x * 0=x$ for $x \in X$

(ii) $(x * y) *(x * z)=z * y \quad$ for $x, y, z \in X$

In $X$ we can define a binary relation $\leq$ by $x \leq y$

if and only if $x * y=0$

\subsection{Proposition}

If $(X, *, 0)$ is a TM-algebra, then i) $x * x=0$;

ii) $(x * y) * x=0 * y$

iii) $x *(x * y)=y$ for any $x, y \in X$

\section{Proof}

i) $x * x=(x * 0) *(x * 0) \quad$ by (i) of definition 2.1

$=0 * 0, \quad$ by (ii) of definition 2.1

$=0$

ii) $(x * y) * x=(x * y) *(x * 0) \quad$ by (i) of definition 2.1

$=0 * y \quad$ by (ii) of definition 2.1

iii) $x *(x * y)=(x * 0) *(x * y) \quad$ by (i) of definition 2.1

$=y * 0 \quad$ by (ii) of definition 2.1

$=y \quad$ by (i) of definition 2.1

\subsection{Proposition}

Let $(X, *, 0)$ be a TM-algebra. Then

$(x * y) * z=(x * z) * y$ for any $x, y, z \in X$ 


\section{Proof}

Given $(X, *, 0)$ is a TM- algebra. Then

$(x * y) *(x * z)=z * y$.

Put $z=x * y$ and $y=z$ in $(2.3 .1)$. Then

$(x * y) * z=(x * z) *(x *(x * y))$

$$
=(x * z) * y, \quad \text { by (iii) of proposition } 2.2
$$

\subsection{Proposition}

Let $(X, *, O)$ be a TM-algebra. Then for any $x, y, z \in X$

i) $x * 0=0 \Rightarrow x=0$;

ii) $(x * z) *(y * z) \leq x * y$

iii) $x \leq y \Rightarrow x * z \leq y * z$ and $z * y \leq z * x$

iv) $x *(x *(x * y))=x * y$

v) $0 *(x * y)=y * x=(0 * x) *(0 * y)$

vi) $(x *(x * y)) * y=0$;

vi) If $x * y=0, y * x=0$ then $x=y$

\section{Proof}

i) $x * 0=x$, by (i) of definition 2.1 .

If $x * 0=0$, then $x=0$, proves the result.

ii) $((x * z) *(y * z)) *(x * y)$

$$
\begin{array}{lr}
=((x * z) *(x * y)) *(y * z) & \text { by proposition } 2.3 \\
=(y * z) *(y * z) & \text { by (ii) of definition } 2.1 \\
=0 & \text { by proposition } 2.2
\end{array}
$$

Hence $(x * z) *(y * z) \leq x * y$.

iii) $x \leq y \Rightarrow x * y=0$.

To prove $x * z \leq y * z$.

That is to prove $(x * z) *(y * z)=0$.

By (ii) of this proposition , $(x * z) *(y * z) \leq x * y$

Since $x * y=0,(x * z) *(y * z)=0$.

Similarly, $(z * y) *(z * x)=0$.

Hence (iii) follows. iv) $x *(x *(x * y))=(x * 0) *(x *(x * y))$

by (i) of definition 2.1

$$
=(x * y) * 0
$$

by (ii) of definition 2.1

$$
=x * y
$$

by (i) of definition 2.1

v) $0 *(x * y)=(x * x) *(x * y)$

by proposition 2.2

$$
=y * x
$$

by (ii) of definition 2.1

$$
=(0 * x) *(0 * y)
$$

by (ii) of definition 2.1

vi) $(x *(x * y)) * y=(x * y) *(x * y)$

by proposition

2.3

$$
=0
$$

by ( iii ) of

proposition 2.2

$$
\begin{aligned}
& \text { vii) } x=x * 0=x *(x * y)=y \quad \text { (or) } \\
& y=y * 0=y *(y * x)=x .
\end{aligned}
$$

Recently, Ahn \& Kim introduced the notion of QS-algebras.

A QS-algebra is obviously a TM-algebra, But a TM- algebra is said to be a QS-algebra if it satisfies the additional relations,

$(x * y) * z=(x * z) * y$ and $y * z=z * y$ for all $x, y, z \in X$.

\subsection{Example}

Let $Z$ be the set of all integers, and let $n Z=\{n x: x \in Z\}, n \in Z$. Then $(Z,-, 0)$ and $(n Z,-, 0)$ are TM-algebras (where "--" is the usual subtraction).

\section{Solution}

$x-0=x$ for all $x \in Z$, and

$(x-y)-(x-z)=z-y$, for all $x, y, z \in Z$.

Hence $(Z,-, 0)$ is a TM -algebra.

Similarly we prove $(n Z,-, 0)$ is a TM-algebra.

\subsection{Example}

Let $X=\{0,1,2,3\}$ be a set with cayley table (Table 1 ). 


\section{Table 1}

\begin{tabular}{c|cccc}
$*$ & 0 & 1 & 2 & 3 \\
\hline 0 & 0 & 1 & 2 & 3 \\
1 & 1 & 0 & 3 & 2 \\
2 & 2 & 3 & 0 & 1 \\
3 & 3 & 2 & 1 & 0
\end{tabular}

Then $(X, *, 0)$ is a TM-algebra.

The relations between TM-Algebra and other algebras are investigated and presented below.

\subsection{Theorem}

Every BCK-algebra is a TM-algebra but the converse is not true.

The above example in 2.6 is a TM-algebra but not BCK algebra since $0 * x \neq 0$ for all $x=1,2,3$.

\subsection{Theorem}

Every TM -algebra is a BH - algebra, but the converse is not true. Similarly, every TM-algebra is a $Q$ algebra, but the converse is not true, as shown in Table 2. Let, $X=\{0,1,2,3\}$

\section{Table 2}

\begin{tabular}{c|cccc}
$*$ & 0 & 1 & 2 & 3 \\
\hline 0 & 0 & 0 & 0 & 0 \\
1 & 1 & 0 & 0 & 0 \\
2 & 2 & 0 & 0 & 0 \\
3 & 3 & 3 & 3 & 0
\end{tabular}

Note that $(X, *, 0)$ is a Q-algebra.

The condition $(x * y) *(x * z)=z * y$ is not satisfied as $(1 * 2) *(1 * 3)=0 * 0=0 \neq 3=3 * 2$.

\subsection{Theorem}

Every TM-algebra is a BCH-algebra. Every $\mathrm{BCH}$ algebra satisfying $(x * y) *(x * z)=z * y$ is a TM-algebra.

\subsection{Theorem}

Every TM-algebra is a BCI-algebra.

\subsection{Theorem}

Every TM-algebra $X$ satisfying $x * z=z$ is a trivial algebra.

\section{Proof}

Put $x=z$ in $x * z=z$, then $z * z=z$ which implies $0=z$

Hence $X$ is a trivial algebra.

\subsection{Lemma}

Let $(X, * 0)$ be a TM-algebra. Then $a * b=a * c$ for all $a, b, c \in X$ implies $0 * b=0 * c$.

\section{Proof}

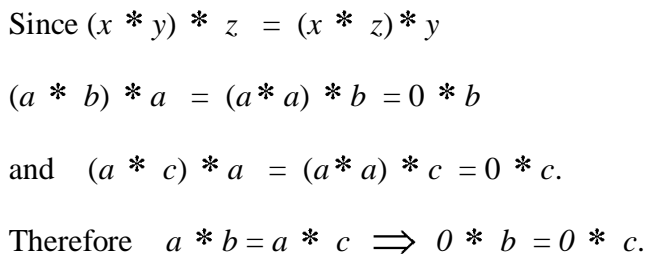

\subsection{Definition}

Define $\boldsymbol{G}(\boldsymbol{X})=\{x \in X: 0 * x=x\}$.

For any TM- $\operatorname{algebra}(X, *, 0)$ the set $\boldsymbol{B}(X)=\{x \in X: 0 \leq x\}$ is called the $p$-radical of $X$. If $B(X)=\{0\}$, then $X$ is said to be a $\boldsymbol{p}$-semisimple TM-algebra.

Note that $G(X) \cap B(X)=\{0\}$

\subsection{Proposition.}

$X$ is p-semisimple if and only if $0 *(0 * x)=x$ for all $x \in X$.

\section{Proof}

Let $X$ be $p$-semisimple. Then $B(X)=\{0\}$.

That is $0 * 0=0$ and $0 * x \neq 0$ for all $x \neq 0$.

Now, $0 *(0 * \mathrm{x})=(0 * 0) *(0 * x)=x * 0=x$.

Conversely, let $0 *(0 * x)=x$. 
Then $0 *(0 * x)=x$ implies $0 * x \neq 0$.

If $0 * x=0$ for $x \neq 0$, then $0 * 0=x$ which implies $x=0$.

Hence $X$ is $p$-semisimple.

\subsection{Proposition}

If $(x, *, 0)$ is a TM-algebra and $x, y \in X$ then

$y \in B(X) \Leftrightarrow(x * y) * x=0$.

\section{Proof}

Let $y \in B(X)$. Then $0 * y=0$.

Now $(x * y) * x=(x * y) *(x * 0)$

$$
\begin{aligned}
& =0 * y \\
& =0, \quad \text { since } y \in B(X) .
\end{aligned}
$$$$
\text { by (ii) of definition } 2.1
$$

Conversely,

$$
\text { let } \begin{aligned}
(x * y) & * x=0 . \text { Then } \\
0 & =(x * y) * x \\
& =(x * y) *(x * 0) \\
& =0 * y \\
& \Rightarrow y \in B(X) .
\end{aligned}
$$

\subsection{Proposition.}

Let $(X, *, 0)$, be a TM-Algebra. If $G(X)=X$, then $X$

is p-semi-simple.

\section{Proof}

Let $G(X)=X . \quad$ Also $G(X) \cap B(X)=\{0\}$.

So $X \cap B(X)=\{0\}$. That is $B(X)=\{0\}$.

Hence $X$ is $\mathrm{P}$-semi simple.

Note that a TM-Algebra in which $G(X)=X$ is a d-algebra.

\subsection{Definition}

Let $(X, *, 0)$ be a TM-algebra. A non-empty subset $I$ of $X$ is called an ideal of $X$ if it satisfies

i) $0 \in I$

ii) $x * y \in I$ and $y \in I$ imply $x \in I$ for all $x, y \in X$.
Any ideal $I$ has the property that $y \in I$ and $x \leq y$ imply $x \in I$.

\section{Table 3}

\begin{tabular}{c|cccc}
$*$ & 0 & $\mathrm{a}$ & $\mathrm{b}$ & $\mathrm{c}$ \\
\hline 0 & 0 & 0 & $\mathrm{c}$ & $\mathrm{b}$ \\
$\mathrm{a}$ & $\mathrm{a}$ & 0 & $\mathrm{c}$ & $\mathrm{b}$ \\
$\mathrm{b}$ & $\mathrm{b}$ & $\mathrm{b}$ & 0 & $\mathrm{c}$ \\
$\mathrm{c}$ & $\mathrm{c}$ & $\mathrm{c}$ & $\mathrm{b}$ & 0
\end{tabular}

Then the set $I=\{0, a\}$ is an ideal of $X$.

\subsection{Proposition}

Let $(X, *, 0)$ be a TM-algebra. Then $B(X)$ is an ideal of $X$.

\section{Proof}

Since $0 *(0 * 0)=0,0 \in \mathrm{B}(\mathrm{X})$.

Let $x * y \in B(X)$ and $y \in B(X)$, then

$0 *(x * y)=0$ and $0 * y=0$

Now, by proposition (2.14) y $\in B(X)$ implies $(x * y) * x=0$

and $\quad x * y \in B(X)$ implies $(x *(x * y)) * x=0$.

$$
\begin{aligned}
& \Rightarrow(x *(x * y)) *(x * 0)=0 \\
& \Rightarrow 0 *(x * y)=0 \\
& \Rightarrow(0 * x) *(0 * y)=0 \\
& \Rightarrow(0 * x) * 0=0 \\
& \Rightarrow(0 * 0) * x=0 \\
& \Rightarrow 0 * x=0 \\
& \Rightarrow x \in B(X) .
\end{aligned}
$$

Therefore $B(X)$ is an ideal of $X$.

\subsection{Definition}

A non-empty subset $S$ of a TM-algebra $(X, *, 0)$ is said to be a sub-algebra of $X$ if $x * y \in S$ whenever $x, y \in S$.

\subsection{Definition}

An ideal $A$ of a TM-algebra $(X, *, 0)$ is said to be 
closed if $0 * x \in A$ for all $x \in A$

\subsection{Proposition}

Every closed ideal of a TM-algebra is a TM- subalgebra.

\section{Proof}

Let $A$ be a closed ideal of a TM-algebra $(X *, 0)$.

Let $x, y \in A$ Then

$0 * x, 0 * y \in A$.

As $0 *(x * y)=(0 * x) *(0 * y), 0 *(x * y) \in A$.

Hence $x * y \in A$. So $A$ is a sub-algebra of TM-algebra.

Note that the converse of the above proposition is not true.

\subsection{Definition}

An ideal $A$ of a TM-algebra $(X, *, 0)$ is said to be translational ideal of $X$ if whenever $x * y \in A, y * x \in A$, then $(x * z) *(y * z) \in A$ and $(z * x) *(z * y) \in A$ for all $x$, $y, z \in X$.

\subsection{Proposition}

If $S$ is a sub algebra of a TM-algebra $(X *, 0)$ then $G(X) \cap S=G(S)$.

\section{Proof}

Obviously $\mathrm{G}(X) \cap S \subseteq G(S)$.

We know $G(S)=\{x \in S \subseteq X: 0 * x=x\}$

Let $x \in G(S)$. Then $0 * x=x$ and $x \in S \subseteq X$ which implies $x \in G(X) \cap S$

Hence $G(S) \subseteq G(X) \cap S$, which proves the result.

\subsection{Theorem}

If $(X, *, 0)$ is a TM-algebra of order 3 , then

i) $G(X) \neq X$

ii) $G(X)$ is an ideal of $X$ if $|G(X)|=1$.

\section{Proof}

i) Let $X=\{0, a, b\}$ be a TM-algebra.

Assume $G(X)=X$.

Then $0 * 0=0, \quad 0 * a=\mathrm{a}, 0 * b=b$.

Also we know $x * x=0$ and $x * 0=x$, for all $x \in X$

Therefore $a * 0=a, a * a=0$ and $b * b=0$.

Let $a * b=0$. It is argued for $b * a=0, a, b$.

Now if $b * a=0$, then $a * b=0=b * a$ and

$(a * b) * a=(a * b) *(a * 0)=0 * b=b$ and

$(b * a) * a=0 * a=a$.

Since $(a * b) * a=(b * a) * a$, it follows that $a=b$, a contradiction.

So $b * a \neq 0$

If $b * a=a$, then

$$
\begin{aligned}
a=b * a & =(0 * b) * a \\
& =(0 * a) * b \\
& =a * b \\
& =0, \quad \text { a contradiction. }
\end{aligned}
$$

If $b * a=b$, then

$$
\begin{aligned}
b=b * a & =(0 * \boldsymbol{b}) * a \\
& =(0 * a) * b \\
& =a * b \\
& =0, \quad \text { a contradiction. }
\end{aligned}
$$

Next if $a * b=a$, then

$(a *(a * b)) * b=(a * a) * b=0 * b=b \neq 0$

contradicting ( $v i$ ) of proposition (2.4)

Finally, let $a * b=b$.

It is argued for $b * a=0, a, b$.

If $b * a=0$, then $b=a * b$

$$
\begin{aligned}
& =(0 * a) * b \\
& =(0 * b) * a \\
& =b * a
\end{aligned}
$$


$=0$, a contradiction

If $b * a=a$, then

$$
\begin{aligned}
b & =a * b \\
& =(0 * a) * b \\
& =(0 * b) * a \\
& =b * a \\
& =a, \text { a contradiction. }
\end{aligned}
$$

If $b * a=b$, then

$$
\begin{aligned}
a & =0 * a \\
& =(b * b) * a \\
& =(b * a) * b \\
& =b * b
\end{aligned}
$$

0 , a contradiction.

Thus it is concluded that there exist some other elements in $G(X)$, which is not in $X$.

(ii) Let $X=\{0, a, b\}$ be a TM-algebra of order 3 .

If the order of $G(X)$ is 1 , then

$G(X)=\{0\}$, is the trivial ideal of $X$.

Conversely,

assume $G(X)$ is an ideal of $X$.

By (i) of this proposition

$|G(X)|=1 \quad$ or $|G(X)|=2$.

Suppose $|G(X)|=2$. Then

$G(X)=\{0, a\}$ or $G(X)=\{0, b\}$.

If $G(X)=\{0, a\}$, since $G(X)$ is an ideal of $X$,

$b * a \notin G(X)$ so $b * a=b$.

Now, $a=0 * a$

$$
\begin{aligned}
& =(b * b) * a=(b * a) * b \\
& =b * b \\
& =0, \text { a contradiction } .
\end{aligned}
$$

Hence $|G(X)| \neq 2$ and so $|G(X)|=1$.

\subsection{Definition}

Let $(X, *, 0)$ and $\left(Y, \Delta, 0^{1}\right)$ be TM-algebras. A mapping $f: X \rightarrow Y$ is called a homomorphism if

$$
\mathrm{f}(x * y)=f(x) \Delta f(y) \text { for all } x, y \in X .
$$

Note that $f^{-1}(Y)=\{x \in X: f(x)=y$ for some $y \in Y\}$ and $f(X)=\{f(x): x \in X\}$ is called the image of $f$.

\subsection{Proposition}

Suppose $f: X \rightarrow Y$ is a homomorphism of TMalgebras. Then

(i) $f(0)=0^{1}$

(ii) If $x * y=0$, for all $x, y \in X$ then $f(x) \Delta f(y)=0^{1}$.

\section{Proof}

$\mathrm{f}(0)=f(0 * 0)=f(0) \Delta f(0)=0^{1}$.

ii) Let $x, y \in X$ and $x * y=0$. Then

$f(x) \Delta f(y)=f\left(x^{*} y\right)=f(0)=0^{1}$.

\subsection{Theorem}

Let $(X, *, 0),\left(Y, \Delta, 0^{\prime}\right)$ be TM-algebras and let $B$ be an ideal of $Y$. Let $f: X \rightarrow Y$ be a homomorphism. Then $f^{-1}(B)$ is an ideal of $X$.

\section{Proof}

We know $f^{--1}(B)=\{x \in X: f(x)=y$ for $y \in B\}$.

Since $0^{1} \in B$ and $f(0)=0^{1}, 0 \in f^{-1}(B)$.

Assume $x * y \in f^{-1}(B)$ and $y \in f^{--1}(B)$, then $f(x * y) \in B$ and $f(y) \in B$.

Since $f$ is a homomorphism, $f\left(x^{*} y\right)=f(x) \Delta f(y) \in \mathrm{B}$

Since $B$ is an ideal of $Y, f(x) \in B$, so $x \in f^{-1}(B)$.

Hence $f^{-1}(B)$ is an ideal of $X$.

\subsection{Definition}

Let $(X, *, 0),\left(Y, \Delta, 0^{\prime}\right)$ be TM-algebras. Let $f: X \rightarrow Y$ 
be a homomorphism. Then the set $\left\{x \in X: f(x)=0^{1}\right\}$ is called the kernel of $\boldsymbol{f}$ and is denoted by $\operatorname{ker} f$.

\subsection{Theorem}

Let $f: X \rightarrow Y$ is a homomorphism of TM-algebras.

Then ker $f$ is an ideal of $X$.

\section{Proof}

Obviously $0 \in$ kerf, since $f(0)=0^{1}$.

Let $x * y \in \operatorname{ker} f$, and $y \in \operatorname{kerf}$.

So $f(x * y)=0^{1}$ and $f(y)=0^{1}$.

That is $f(x) \Delta f(y)=0^{1}$

$$
\begin{aligned}
& \Rightarrow f(x) \Delta 0^{1}=0^{1} \\
& \Rightarrow f(x)=0^{1}
\end{aligned}
$$

Hence $x \in \operatorname{ker} f$,

So $\operatorname{ker} f$ is an ideal of $X$.

\section{CONCLUSION}

Concept of TM-algebra proposed, in the present work, has been evaluated against well established algebraic theorems. It has been observed that the TM-algebra satisfy the various conditions stated in the $\mathrm{Q} / \mathrm{BCH} / \mathrm{BCI} / \mathrm{BCK}$ algebras and can be considered as the generalization of all these algebras.

\section{REFERENCES}

[1] J. Neggers, S. S. Ahn and H. S. Kim., On d-algebras, Math. Slovaca 49 (1999), 9-26

[2] J. Neggers, S. S. Ahn and H. S. Kim. On Q-algebras, IJMMS 27 (2001), 749-757.

[3] K. Iseki, On BCI -algebras, Math. Sem. Notes Kobe Univ. 8 (1980), 25- 130.

[4] K.Iseki and S.Tanaka. An introduction to the theory of BCk- algebras, Math. Japon. 23 (1978), 1-26

[5] Q.P. Hu and X. Li. On BCH-algebras, Math. Sem. Notes Kobe Univ. 2 (1983), 313-320.

[6] Q.P. Hu and X. Li. On proper BCH-algebras, Math. Japon. 30 (1985), 659- 661.

[7] Y.B. Jun, E.H. Roh and H.S. Kim. On BH - algebras, Sci. Math 1 (1998), 347-354.

[8] S.S. Ahn and H.S. Kim, On QS -algebras, J. Changcheong Math. Soc. 12 (1999), 33-41 